\title{
Towards the Applicability of the Modified von Kármán Spectrum to Predict Trailing Edge Noise
}

\author{
Marcus Bauer ${ }^{1}$ and Andreas Zeibig ${ }^{2}$ \\ ${ }^{1}$ Institute of Acoustics and Speech Communication, \\ Dresden University of Technology, 01062 Dresden, Germany \\ Present address: DLR (German Aerospace Center), Institute of Aerodynamics \\ and Flow Technology, Lilienthalplatz 7, 38108 Braunschweig, Germany \\ Marcus.Bauer@dlr.de \\ 2 Institute of Acoustics and Speech Communication, \\ Dresden University of Technology, 01062 Dresden, Germany \\ Andreas.Zeibig@ias.et.tu-dresden.de
}

\begin{abstract}
Summary
Trailing edge noise can be predicted with the help of a synthetic turbulent velocity field. Assuming isotropic conditions this field may be generated via an energy spectrum of turbulence like the modified von Kármán spectrum (MVKS). In this work one-dimensional wavenumber spectra of turbulence obtained from hot-wire measurements at the trailing edges of a thin, flat plate and a NACA0012 airfoil were compared to the respective spectra extracted from the MVKS. Good agreement at all measuring positions is possible with a modified form of the MVKS. The remaining discrepancies can be attributed to the anisotropy of boundary layer turbulence.
\end{abstract}

\section{Introduction}

Airframe noise is an important part of an aircraft's landing approach noise. In order to design quieter aircraft there is a strong demand for tools that allow a computational optimisation of its respective components, e.g. landing gears, slats and flaps. Unfortunately this is hardly possible with present computer resources via a Direct Numerical Simulation (DNS) solving the full governing flow equations (Navier Stokes Equations, NSE). This has given rise to the application of a hybrid $\mathrm{CFD}^{1} / \mathrm{CAA}^{2}$ approach based on simplified forms of the NSE and on a synthetic stochastic isotropic turbulent velocity field [7]. With this means broadband trailing edge noise, a significant contribution to

\footnotetext{
${ }^{1}$ Computational Fluid Dynamics

${ }^{2}$ Computational Aeroacoustics
} 
airframe noise, has successfully been computed $[2,5]$. The synthetic velocity field is based on the assumption of the modified von Kármán spectrum (MVKS) as the prevailing energy spectrum of turbulence [3, 9, 10, 11].

The aim of the present investigations was to find out to what extent the MVKS is appropriate to describe turbulent trailing edge flows. Therefore one-dimensional turbulent wavenumber spectra obtained from hot-wire measurements at the trailing edges of two test objects were compared to the respective spectra extracted from the MVKS. Please note that the topic of this paper is not to present trailing edge noise results computed with the hybrid approach (please refer to references $[2,5]$ in case of interest). Consequently the approach will only be described briefly in so far as the MVKS is concerned.

\section{Procedure of the Studies}

\subsection{Coordinate System and Measurements}

Figure 1 shows the coordinate system. Test objects were a NACA0012 airfoil (dimensions $\left.L_{x}=120 \mathrm{~mm}, L_{z}=140 \mathrm{~mm}\right)$ and a thin, flat plate $\left(L_{x}=\right.$ $200 \mathrm{~mm}, L_{y}=0.3 \mathrm{~mm}, L_{z}=100 \mathrm{~mm}$ ). In both cases the angle of attack was zero degrees and the incoming flow velocity $v_{0 x}=37.5 \mathrm{~m} / \mathrm{s}$, corresponding to a Mach-number $M a=0.11$. The measurements were carried out in the aeroacoustic wind-tunnel of the Institute of Acoustics and Speech Communication at Dresden University of Technology using a triple sensor hot-wire probe. It was positioned with a lightweight traverse system of accuracy \pm 0.01 $\mathrm{mm}$ in every coordinate direction. At both objects time responses of the velocity vector components $\boldsymbol{v}_{i}(i=x, y, z)$ were recorded in approximately 40 $y$-coordinates at $z=0$ as close as possible to the trailing edge in $x$-direction $(x \approx 1 \mathrm{~mm}) .16,384=2^{14}$ samples were recorded at a sampling frequency of $25 \mathrm{kHz}$ (plate) and $250 \mathrm{kHz}$ (airfoil) using anti-aliasing low-pass filters with cut-off frequencies of $10 \mathrm{kHz}$ and $100 \mathrm{kHz}$, respectively. The boundary layer was tripped with a tape of height $0.3 \mathrm{~mm}$ and length $1.2 \mathrm{~mm}$ on both sides of the objects. At the flat plate this tape was attached $6 \mathrm{~mm}$ downstream of the leading edge and at the NACA0012 airfoil at the $x$-coordinate of maximum profile thickness, respectively.

\subsection{The MVKS in the Framework of the Hybrid Approach}

The hybrid approach used in $[2,5]$ to compute broadband trailing edge noise is based on splitting the field variables into a time averaged and a generic fluctuating part. In the first step the mean flow field is calculated with DLR's CFD-Code FLOWer as a solution of the RANS ${ }^{3}$-equations using the standard Wilcox $k-\omega$ turbulence model [12], $k$ denoting the turbulent kinetic energy

\footnotetext{
${ }^{3}$ Reynolds Averaged Navier Stokes
} 
and $\omega$ its specific dissipation rate, respectively. In the second step a modified form of the $\mathrm{LEE}^{4}$ with a source term is solved with DLR's CAA-Code PIANO to compute the time-dependent variables. The source term is calculated from a frozen synthetic turbulent velocity field $\boldsymbol{v}_{s t}$ which is given by a sum of discrete Fourier modes [7].

Generating $\boldsymbol{v}_{s t}$ requires a turbulent energy spectrum $E(\alpha)$, $\alpha=\sqrt{\alpha_{x}^{2}+\alpha_{y}^{2}+\alpha_{z}^{2}}$ denoting the wavenumber. At present $E(\alpha)$ is modelled by the MVKS:

$$
E(\alpha)=C_{1} \frac{2 / 3 k}{\alpha_{e}} \frac{\left(\frac{\alpha}{\alpha_{e}}\right)^{4}}{\left(1+\left(\frac{\alpha}{\alpha_{e}}\right)^{2}\right)^{17 / 6}} \mathrm{e}^{-C_{2}\left(\frac{\alpha}{\alpha_{\nu}}\right)^{2}} .
$$

It has its maximum at wave number $\alpha_{e}$ and reaches up to the Kolmogorov wave number $\alpha_{\nu}$. Both $\alpha_{e}$ and $\alpha_{\nu}$, and thus the whole spectrum, can be calculated from the RANS-results of $k$ and $\omega$. The parameter $C_{2}$ in the exponent is $C_{2}=2$ and the scaling factor $C_{1}$ ensures that

$$
\int_{0}^{\infty} E(\alpha) \mathrm{d} \alpha=k
$$

Here $C_{1}$ is (for every set of input parameters $k$ and $\omega$ ) calculated as the ratio of $k$ and the integral over $E(\alpha)$, which is computed numerically with the trapezoidal rule (for $C_{1}=1$ ). Note that turbulence can only be described by an energy spectrum $E(\alpha)$ if isotropy is assumed.

\subsection{Characterising the Applicability of the MVKS}

One-dimensional wavenumber spectra $\Phi_{x x}\left(\alpha_{x}\right), \Phi_{y y}\left(\alpha_{x}\right)$ and $\Phi_{z z}\left(\alpha_{x}\right)$ obtained from measurement as well as from (1) were compared to each other, see Figure 2. $E(\alpha)$ given by the MVKS was transformed into these longitudinal $\left(\Phi_{x x}\right)$ and lateral $\left(\Phi_{y y}, \Phi_{z z}\right)$ one-dimensional spectra via [1]:

$$
\begin{gathered}
\Phi_{x x}\left(\alpha_{x}\right)=\frac{1}{2} \int_{\alpha_{x}}^{\infty} \frac{E(\alpha)}{\alpha}\left(1-\frac{\alpha_{x}^{2}}{\alpha^{2}}\right) \mathrm{d} \alpha, \\
\Phi_{y y / z z}\left(\alpha_{x}\right)=\frac{1}{4} \int_{\alpha_{x}}^{\infty} \frac{E(\alpha)}{\alpha}\left(1+\frac{\alpha_{x}^{2}}{\alpha^{2}}\right) \mathrm{d} \alpha .
\end{gathered}
$$

\subsection{Generation of the MVKS}

The parameter $C_{2}$ governs the decrease of the MVKS at high wavenumbers. To provide for a better agreement with the one-dimensional wavenumber

\footnotetext{
${ }^{4}$ Linearized Euler Equations
} 
spectra $\Phi_{i i}\left(\alpha_{x}\right)$ from measurement modified forms of the MVKS with $C_{2} \neq 2$ were used in this study, too, see below.

Furthermore the input parameters $k$ and $\omega$ were not taken from the RANS computation here. Actually, $k$ was calculated from the measured $v_{i}(\boldsymbol{x}, t)$ at coordinate $\boldsymbol{x}=[x, y, z]^{\mathrm{T}}$ via

$$
k=\frac{1}{2}\left(\overline{v_{x}(\boldsymbol{x}, t)^{2}}+\overline{v_{y}(\boldsymbol{x}, t)^{2}}+\overline{v_{z}(\boldsymbol{x}, t)^{2}}\right),
$$

the overline denoting the time-average. The value of $\omega$ was estimated to provide for a best possible coincidence of the $\Phi_{i i}\left(\alpha_{x}\right)$ from measurement and theory. If $k$ and $\omega$ would have been taken from the RANS-solution, the discrepancy of these spectra would have increased due to RANS inaccuracies, see below. However, the main goal of this study was not to assess the applicability of the input parameters $k$ and $\omega$ rather than that of the MVKS itself.

\section{Results and Discussion}

\subsection{Wavenumber Spectra of Trailing Edge Turbulence}

Figure 3 compares one-dimensional wavenumber spectra $\Phi_{i i}\left(\alpha_{x}\right)$ obtained from the MVKS and from a measurement at the NACA0012 airfoil at $y \approx$ $-1.6 \mathrm{~mm}$. Due to the limited signal-to-noise ratio of the hot-wire measurement technique no values of $\Phi_{i i}\left(\alpha_{x}\right)$ below approximately $10^{-5} \mathrm{~m}^{3} / \mathrm{s}^{2}$ could be resolved. Using the generic form of the MVKS with $C_{2}=2\left(\Rightarrow C_{1}=1.69\right.$ for the employed values of $k$ and $\omega$ ) there is a good agreement at wavenumbers $\alpha_{x}<2.0 \cdot 10^{3} / \mathrm{m}$ but a rather poor agreement at higher wavenumbers. A modified form with $C_{2}=50\left(C_{1}=2.24\right)$ provides for a good coincidence throughout all $\alpha_{x}$. The remaining deviations can be attributed to the anisotropy of boundary layer turbulence $[4,6,8]$. They can not be overcome by another $E(\alpha)$ spectrum as for example in Figure $3 \Phi_{x x}\left(\alpha_{x}\right)$ from measurement falls below $\Phi_{x x}\left(\alpha_{x}\right)$ from the MVKS while at the same time $\Phi_{y y}\left(\alpha_{x}\right)$ and $\Phi_{z z}\left(\alpha_{x}\right)$ from measurement exceed the respective spectra extracted from the MVKS.

Figure 4 shows results from the flat plate at $y \approx-0.7 \mathrm{~mm}$. There is a satisfying agreement of the onedimensional wavenumber spectra from measurement and the generic MVKS throughout all wavenumbers. The remaining discrepancies are again due to anisotropy. The upper cut-off frequency of the measurement underlying Figure 4 is lower than in Figure 3 because as mentioned above the sampling frequency at the flat plate was only $25 \mathrm{kHz}$ instead of $250 \mathrm{kHz}$ as in the NACA0012 measurement. The spurious peaks in the measured spectra at wavenumbers $\alpha_{x}<100 / \mathrm{m}$ are most problably due to low-frequency electromagnetic disturbance (e.g. PC-monitor) of the hot-wire sensor signals. 
At both objects the measured spectra confirm that the share of the small eddies increases when the coordinate $y=0$ is approached [6], i.e., the $\Phi_{i i}\left(\alpha_{x}\right)$ spectra decay slower towards higher wavenumbers as $y \rightarrow 0$. Considering similar $y$-coordinates, however, the share of the small eddies at the flat plate is always higher than at the NACA0012 airfoil. This leads to the following consequences:

- Flat plate: There is a good agreement of the $\Phi_{i i}\left(\alpha_{x}\right)$ from measurement and from the generic MVKS for larger values of $y$, cp. Figure 4 , but as $y \rightarrow 0$ the $\Phi_{i i}\left(\alpha_{x}\right)$ from measurement exceed those from the MVKS at high wavenumbers.

- NACA0012: The $\Phi_{i i}\left(\alpha_{x}\right)$ spectra from measurement fall below those of the generic MVKS at high wavenumbers in case of large $y$, cp. Figure 3, but on the other hand there is a good agreement as $y \rightarrow 0$.

\subsection{Trailing Edge RANS Results}

Next to a correct formulation of the turbulent energy spectrum $E(\alpha)$ itself reliable values of $k$ and $\omega$ from the RANS-computation are also crucial to generate a proper $E(\alpha)$. In this context Figure 5 illustrates $k(y)$ and $\omega(y)$ from two RANS mean flow solutions for the flat plate. In the first computation its small thickness of only $L_{y}=0.3 \mathrm{~mm}$ was neglected, while in the second it was taken into account. Obviously this results in significant differences in $k$ and $\omega$ as $y \rightarrow 0$. Regarding the finite thickness clearly provides for a better agreement with measured data.

However concerning the agreement of the onedimensional wavenumber spectra from measurement and the MVKS, the remaining RANS inaccuracies may be as important as the choice of the energy spectrum, see the dashed line in Figure 4 where $k$ and $\omega$ were taken from the RANS solution of the flat plate to obtain the MVKS.

\section{Conclusion}

At some coordinates the one-dimensional wavenumber spectra computed from the generic MVKS are in good agreement with respective spectra obtained from hot-wire measurements at the trailing edges of a thin, flat plate and a NACA0012 airfoil. However, in general, modified forms of the MVKS provide for a significant improvement resulting in a good agreement with measured spectra throughout all positions at the trailing edges of both objects. The remaining discrepancies are attributed to the anisotropy of the boundary layer turbulence.

Thus it seems worthwhile to investigate the influence of enhanced turbulence spectra and anisotropy, respectively, on the computed trailing edge noise. Also the influence of the fidelity of the RANS results underlying an energy spectrum of turbulence should be subject of future interest. 


\section{Acknowledgements}

The authors wish to thank the DLR and the DFG (German Research Foundation) for kind funding of this work under grant number KO1242/6 in the framework of the $S W I N G+$ project (Simulation of Wing Flow Noise Generation).

\section{References}

[1] G. K. Batchelor. The Theory of Homogeneous Turbulence. Cambridge at the University Press, 1956.

[2] M. Bauer. Berechnung der Schallabstrahlung überströmter Hinterkanten. Diploma thesis, Institute of Acoustics and Speech Communication, Dresden University of Technology, 2002.

[3] W. Béchara, C. Bailly, P. Lafon, and S. Candel. Stochastic Approach to Noise Modeling for Free Turbulent Flows. AIAA Journal, 32(3):455-463, March 1994.

[4] D. C. Dunn and J. F. Morrison. Anisotropy and Energy Flux in Wall Turbulence. Journal of Fluid Mechanics, 491:353-378, 2003.

[5] R. Ewert and M. Bauer. Towards the Prediction of Trailing Edge Noise via Stochastic Surface Sources. AIAA-paper 2004-2861, American Institute for Aeronautics and Astronautics, 2004.

[6] J. O. Hinze. Turbulence. Mc Graw Hill Book Company, Inc., 1959.

[7] R. H. Kraichnan. Diffusion by a Random Velocity Field. Phys. Fluids, 13(1):22-31, 1970.

[8] J. M. Österlund and A. V. Johansson. Turbulence Statistics of Zero Pressure Gradient Turbulent Boundary Layers. (to be submitted), Dept. of Mechanics, KTH, SE-100 44 Stockholm, Sweden.

[9] Y. H. Pao. Structure of Turbulent Velocity and Scalar Fields at Large Wave Numbers. Phys. Fluids, 8(6):1063-1075, 1965.

[10] P. G. Saffman. On the Fine-Scale Structure of Vector Fields Convected by a Turbulent Fluid. Journal of Fluid Mechanics, 16:545-572, 1963.

[11] T. von Kármán. Progress in the Statistical Theory of Turbulence. Proc. Natl. Acad. Sci. U.S., 34:530-539, 1948.

[12] D. C. Wilcox. Turbulence Modeling for CFD. DCW Industries, Inc., 1993.

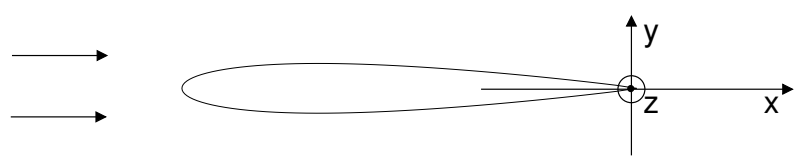

Figure 1 Coordinate system; the origin was centered at the trailing edge in the $y$ and $z$-direction, respectively 


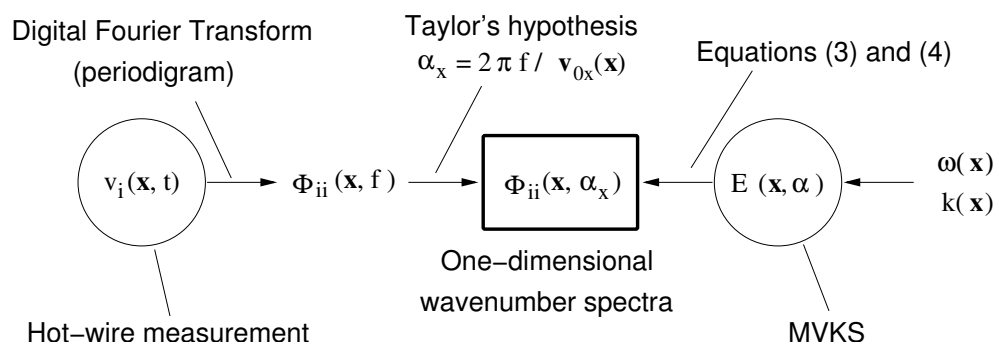

Figure 2 Illustration of the procedure to compare data from the hot-wire measurement to the MVKS; $i=x, y, z$
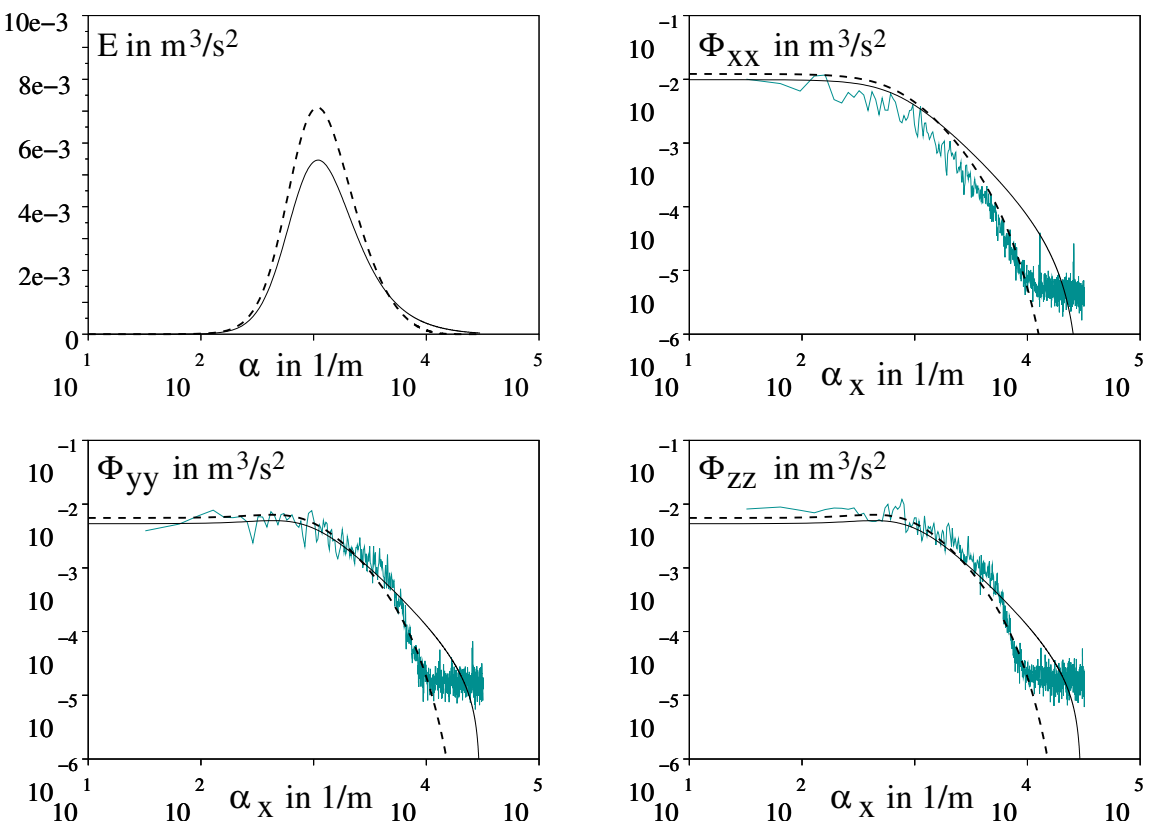

Figure 3 Comparison of one-dimensional spectra $\Phi_{i i}\left(\alpha_{x}\right)$ obtained from a NACA0012 measurement at $\boldsymbol{x}=[1.0 \mathrm{~mm},-1.6 \mathrm{~mm}, 0.0 \mathrm{~mm}]^{\mathrm{T}}$ and from $E(\alpha)$ given by the modified von Kármán spectrum. $E(\alpha)$ was calculated for $k=19.0 \mathrm{~m}^{2} / \mathrm{s}^{2}$ (from measurement) and $\omega=25000 / \mathrm{s}$ (best fit); solid line: $C_{2}=2$, dashed line: $C_{2}=50$ 

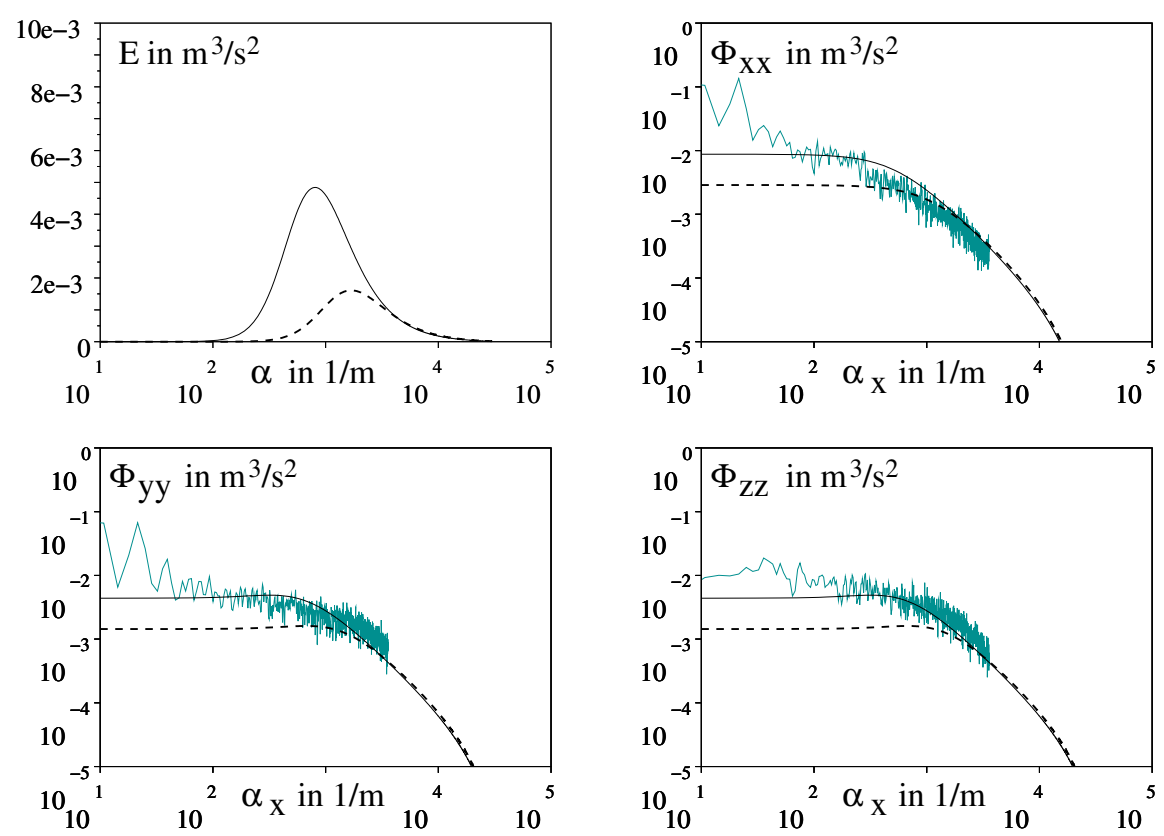

Figure 4 Comparison of one-dimensional spectra $\Phi_{i i}\left(\alpha_{x}\right)$ obtained from a flat plate measurement at $\boldsymbol{x}=[1.0 \mathrm{~mm},-0.7 \mathrm{~mm}, 0.0 \mathrm{~mm}]^{\mathrm{T}}$ and from $E(\alpha)$ given by the generic modified von Kármán spectrum with $C_{2}=2$; solid line: $E(\alpha)$ calculated for $k=12.0 \mathrm{~m}^{2} / \mathrm{s}^{2}$ (from measurement) and $\omega=15000 / \mathrm{s}$ (best fit); dashed line: $E(\alpha)$ calculated from RANS results $\left(L_{y}=0.3 \mathrm{~mm}\right)$ of $k=8.0 \mathrm{~m}^{2} / \mathrm{s}^{2}$ and $\omega=25000 / \mathrm{s}$, compare Figure 5 below
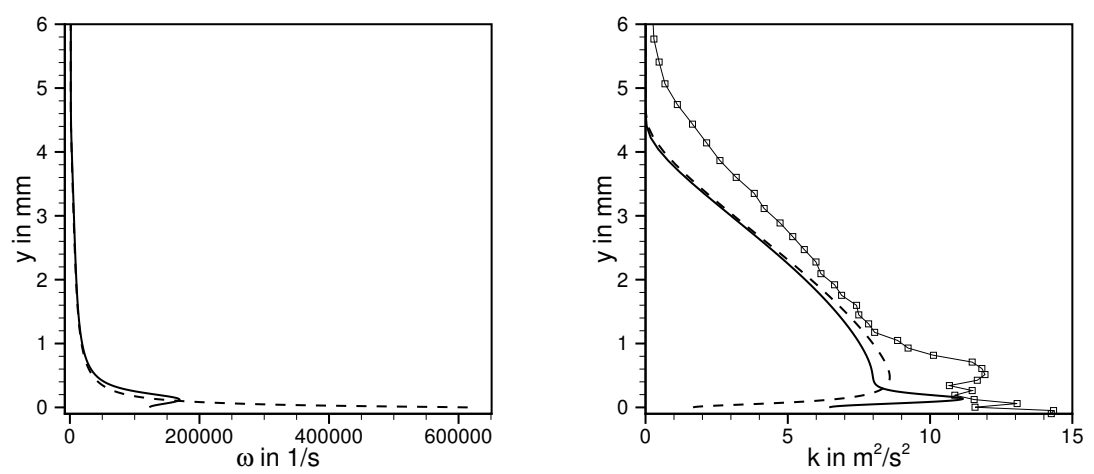

Figure 5 RANS-solutions of $\omega(y)$ and $k(y)$ from the thin, flat plate at $x \approx 1 \mathrm{~mm}$ for $L_{y}=0.0 \mathrm{~mm}$ (dashed lines) and $L_{y}=0.3 \mathrm{~mm}$ (solid lines) plus the respective $k(y)$ from the hot-wire measurement (symbols) 